\section{Elementary particle theories and phenomena}

Particle Interactions at Very High Energies. Edited by David Speiser, Francis Halzen and Jaques Weyers. Part A: Pp. xii +398. \$33.60. Part B: Pp. xiii +366 . $\$ 30.00$. (NATO Advanced Study Institutes Series.)(Plenum:London, 1974.)

THESE two volumes cover the main lectures given at the Summer Institute on Elementary Particle Physics held at Louvain in August 1973. Looking back over the years it is probably fair to say that almost every summer school volume that has appeared has contained at least one noteworthy set of lectures, and the present volumes are no exception. Although both books shelter under the umbrella of general elementary particle physics, they are really very different in content and subject matter, and it is, therefore, best to deal with them separately.

Part A is concerned with hadronic interactions at very high energies, and concentrates mainly upon the phenomenological results that have been flowing in such profusion from the CERN Intersecting Storage Rings (ISR) and,

\section{BOOKS ON PURE AND APPLIED SCIENCE}

\section{Books reviewed or men- tioned in this journal are available from stock.}

Catalogues,on application.

Please state interests.

\section{SCIENTIFIC LIBRARY}

ANNUAL SUBSCRIPTION from $£ 4.00$

Reduced rates for multiple subscriptions Available in U.K. only

Prospectus free on request

\section{H. K. LEWIS \& Co. Ltd. LONDON : 136 GOWER STREET, WCIE 6BS}

Telephone: $01-3874282$ more recently, from the Fermi National Accelerator Laboratory near Chicago. It touches, too, upon the theories and models that have been conjured up to try to interpret the vast amount of data. But if there is a single overwhelming impression left by the reading of this volume it is simply one of amazement and awe; amazement at the absolutely prodigious flow of data from the new machines and awe in the face of the fascinating and complex behaviour of nature at these energies.

There is considerable overlap between several of the lecturers. The most interesting article is Sens', which covers the shadowy area of physics lying between the domain of the high energy physicist and that of the machine engineer: namely the design and structure of the ISR, its beam characteristics, its performance level, its instabilities and their cures, and the connection between the raw measurements and the quantities of physical interest. It is particularly exciting to read of Sens' optimism about the possibility of storing antiprotons in the ISR, using $400-\mathrm{GeV}$ protons from the new CERN Super Proton Synchrotron (SPS) as a source of $25-\mathrm{GeV}$ antiprotons. One awaits with great expectation and some trepidation the results of proton antiproton collisions at ISR energies-many a theory will surely topple.

All three authors concentrate on proton-proton collisions at very high energies, with coverage of elastic reactions, total cross-sections and many body production, the latter in the guise of 'inclusive' reactions. Jacob's treatment of the data is detailed and very wide ranging. Horn applies himself more to "general trends", that is, to the approximate phenomenological rules that seem to summarise the data. Both Jacob and Horn discuss the theoretical background, but neither does so in a sufficiently comprehensive manner to allow the nonexpert to follow. Horn does succeed, though, in giving one a taste of the remarkable success enjoyed by Regge theory, in its extension to many body reactions by Mueller, at least in explaining the gross features of the data.

Finally, there are the notes of Halzen (Model Independent Features of Diffraction) which provide a rather pedantic, and sometimes careless, list of theoretical results that can be derived from 'deep' principles, that is, from axiomatic field theory. Some discussion of their relevance, or lack of relevance, to present day experiments is given.

All in all this is an interesting, though not distinguished, collection of notes the value of which is not a little diminished by the outrageous presence of 18 blank pages.

Part B reminds one of how much easier it is to write elegantly on a purely theoretical topic than on a pnenomenological one. All the lectures are clear; arguments are logically developed; examples reinforce one's understanding, and one emerges feeling that one has been subjected to a valuable pedagogical experience.

Gilman (Deep Inelastic Scattering and Final State Hadrons) gives a good summary of the theory of electronproton and neutrino-proton collisions at large momentum transfer, with a judicious blend of ideas about scaling, partons, the structure of the produced hadrons, and just enough of experiment to motivate the treatment. The article by Weyers (Constituent Quarks and Current Quarks) tackles one of the most tantalising problems in elementary particle theory, namely the relationship between those quarks out of which the hadrons seem to be built, and the quarks in terms of which the weak and electromagnetic currents are simply expressed. There is a useful summary of the pros and cons of the group $\mathrm{U}(6)$ as a classification group for the hadrons, and a good pedagogical introduction to the Melosh transformation. Carlitz (Chiral Symmetry and the Hadron Spectrum) repeats much of the material of Weyers, but less adequately. It is hard to justify the duplication. A united effort by the two authors would surely have been more successful

The great mystery about quarks is their non-appearance as identifiable, individual particles in the real world. Models have been blithely advocated in which the quarks are permanently bound inside the hadrons which they constitute. In his article (Permanently Bound Quarks) Mandula presents a fascinating speculative study of a 'field theory' in which the fundamental quanta cannot materialise as free particles. The picture, though a little contrived does expose the basic and somewhat frightening difficulties.

The other principal article in this volume, a major tour de force that was originally issued as a CERN Yellow Report, is the evocatively titled "Diagrammar" of 't Hooft and Veltman. Inspired by the difficulties of applying the canonical rules of field theory to gauge theories, they have tried to reformulate all the essential rules in terms of a diagrammatic calculus (essentially a sophisticated form of Feynman's propagator approach) and to de-emphasise completely both the role of the field and the Lagrangian. Many of the derivations of standard results, for example, of the Cutkosky rules for discontinuities of Feynman diagrams, are far clearer and simpler than in the conventional treatments. The $n$-dimensional regularisation method invented by the authors, is also discussed in some detail. This is a long article and, generally, is very clear, but it leaves one with the feeling that just a little more effort would have ironed out the few irritating sections in which the reasoning is difficult to follow.

E. Leader 